\title{
When cold atoms meet mesoscopics
}

J.-P. Brantut et al., Conduction of Ultracold Fermions Through a Mesoscopic Channel, 2012, arXiv:1203.1927v2.; Nature 491, 736739 (2012).

\section{Recommended with a Commentary by Thierry Giamarchi, University of Geneva}

In the last decade or so, cold atomic system have shown, by their ability to realize model systems and the control over parameters such as kinetic energy and interactions, that they can be fantastic quantum simulators to tackle questions pertaining to traditional condensed matter systems [1]. In addition they have offered several new and exciting puzzles, in particular concerning out of equilibrium situations, since they are very good realizations of isolated quantum systems. Despite this convergence of interests the methods and possibilities to perform measurements are still widely different in the two systems, and it is not always easy to realize one particular type of probe, very simple in one of the systems, in the other.

This is in particular the case for one quantity that it is practically taken for granted in condensed matter, namely measuring the transport properties of a sample. One situation we are thoroughly familiar with is the case of a system attached to two reservoirs (see Fig. 1(left)) imposing a finite voltage difference, or a temperature difference through the sample. Besides the near equilibrium situation, where one accesses the conductance of the system, such a device is one of the main cases where one can tackle also out of equilibrium physics in a condensed matter context (see e.g. [2] for an interesting case on a quantum wire). On the theoretical front such systems are ideal to connect the conductance to transmission coefficients of the sample for non-interacting systems via LandauerBüttiker approaches and their generalization to the case of interacting systems.

Realizing similar transport measurements in the case of cold atoms is not so trivial, because the whole cloud of particle is kept in the trap by a confining potential growing as $r^{2}$ from the center of the trap. The system is not translationally invariant and stirring current in it is not so easy. A typical workaround for such a problem is either to excite the system at finite frequency, realizing the equivalent of an optical conductivity measurement $[4,5]$, or to shift brutally the center of the trap, therefore giving a momentum kick to the system and then subsequently measuring by how much the system has moved (see e.g. [6] where this method was used for disordered bosons).

Recently the Zurich group found an elegant way to bring the measurements in the cold atom context much closer to the situation we are familiar with in condensed matter, thereby opening many new exciting possibilities. The device is shown on Fig. 1(right). A laser is creating a repulsive potential which is preventing the atoms of a large cloud to enter the center region. One thus realizes quite naturally a system of a film in the central region coupled to two much larger regions that can thus act as reservoirs. The total number of atoms is of the order of $10^{4}$. A magnetic field gradient allows to create an imbalance in the potential, leading to an imbalance of population in the left and right regions. This imbalance of population creates a current in the central part until equal population is restored making this system an excellent equivalent of the corresponding condensed matter device. The current can be measured by monitoring the population of the reservoirs. In the paper [3] ohmic conducting of the central part has been convincingly demonstrated (see Fig. 1).

Of course the device presents a certain number of advantage that offers great possibilities for future studies. Among those:

- As usual in cold atom systems the interacting can be varied. It is thus in particular possible to study strongly interacting systems as well. Note that by this method the interaction is globally 

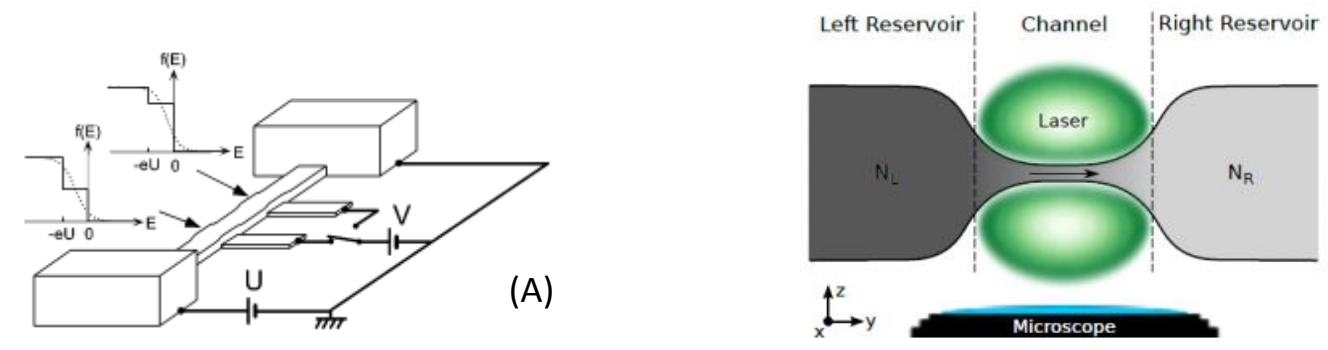

(C)

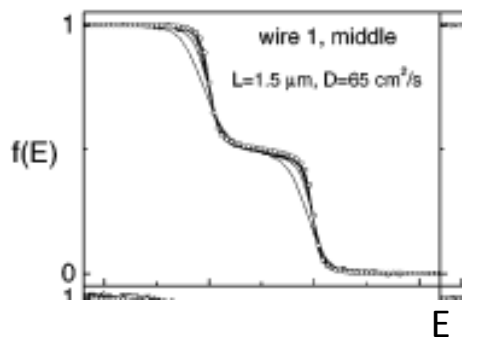

(B)

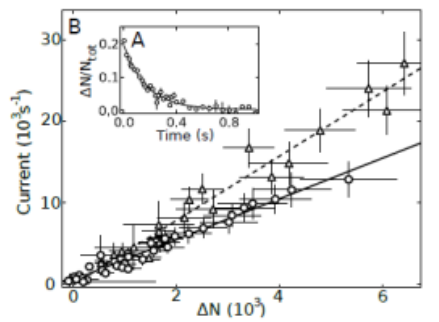

(D)

Figure 1: Two cases of a sample connected to two reservoirs allowing measurement of transport properties. (Left) Condensed matter (from [2]). A) a quantum wire is attached to two different reservoirs at two different voltages. B) the distribution function shows two plateaux, at energies connected to the two voltages, rather than the naive Fermi distribution at a potential interpolating as a function of the distance of the reservoirs between the two voltages. This is the consequence of an out of equilibrium situation. (right) cold atoms (From [3]). C) The corresponding device realizes a thin film connected to two reservoirs, using a laser to repel particles from the central region. D) current as a function of the initial population difference between the reservoirs (insert: population imbalance as a function of time) showing the ohmic behavior of the central region.

varied so that the reservoirs would be interacting as well, a situation slightly different from the traditional condensed matter one for which we usually consider the reservoirs as non-interacting (or at least Fermi liquid like). This difficulty can potentially be circumvented in the future by using an optical lattice as discussed below.

- The temperature of the left and right part can also be imbalanced allowing measurements of thermal transport as well.

- Although the central part is for the moment two dimensional, the use of an optical lattice can allow other geometries as well. For example it would be possible to split the two dimensional film in one dimensional lines by putting a transverse periodic potential created by an optical lattice. Other types of potential can be (and have been) used allowing for example to study the effects of disorder as well.

Some of these possibilities have started to be explored both experimentally and theoretically. On the experimental front, the use of strongly attractive interactions have allowed to turn the fermionic system into a system of pairs, thus allowing to tackle the transport through a two dimensional fluid 
of hard core bosons, which under the right parameters should be a superfluid [7]. The change of transport between the superfluid and normal phase of the fermions has been observed. In another paper disorder has been added [8] to the central region using a speckle pattern to study the impact of the strong disorder on the transport properties. At the moment the effects seem to be largely due to the existence of a percolation threshold allowing or not the bosons (pairs) to propagate from the left reservoir to the right, but with improvement of the system Bose glass type physics will certainly be accessible as well.

Of course the system is not yet perfect. The main drawbacks at the moment are the following

- As with any fermionic system so far in cold atoms the temperature is still quite high (typically around $T_{F} / 10$ where $T_{F}$ is the Fermi temperature. This limits the possibility to probe quantum effects. The situation is better for the case of very large attraction when the fermions are paired.

- The number of atoms in the reservoir is not "infinitely" large which means that the imbalance decreases with time making the time evolution and thus the current more complicated to compute. Of course this situation could also be viewed as an interesting theoretical challenge. On a practical side, the population is however large enough so that reasonable "steady state" results could be inferred from the behavior at short time.

Despite these limitations this device opens a very large number of possibilities to tackle in a controlled way several questions on transport that were previously inaccessible in the cold atom context. It will doubtless be improved in the future and already stimulates a large number of experimental and theoretical studies.

\section{References}

[1] I. Bloch, J. Dalibard, and W. Zwerger, Reviews of Modern Physics 80, 885 (2008).

[2] H. Pothier et al., Physical Review Letters 79, 3490 (1997).

[3] J.-P. Brantut et al., Conduction of Ultracold Fermions Through a Mesoscopic Channel, 2012, arXiv:1203.1927v2.; Nature 491, 736739 (2012).

[4] T. Stöferle et al., Physical Review Letters 92, 130403 (2004).

[5] A. Tokuno and T. Giamarchi, Physical Review Letters 106, 205301 (2011).

[6] M. Pasienski, D. Mckay, M. White, and B. DeMarco, Nature Physics 6, 677 (2010).

[7] D. Stadler et al., Observing the Drop of Resistance in the Flow of a Superfluid Fermi Gas, 2012, arXiv:1210.1426v1.

[8] S. Krinner et al., Superfluidity with disorder in a quantum gas thin film, 2012, arXiv:1211.7272v1. 